\title{
Developing Interpretable Models with Optimized Set Reduction for Identifying
} High Risk Software Components ${ }^{1}$

\author{
Lionel C. Briand, Victor R. Basili and Christopher J. Hetmanski \\ Institute for Advanced Computer Studies, \\ Computer Science Department, \\ University of Maryland, College Park, MD, 20742
}

\begin{abstract}
Applying equal testing and verification effort to all parts of a software system is not very efficient, especially when resources are limited and scheduling is tight. Therefore, one needs to be able to differentiate low / high fault frequency components so that testing / verification effort can be concentrated where needed. Such a strategy is expected to detect more faults and thus improve the resulting reliability of the overall system. This paper presents the Optimized Set Reduction approach for constructing such models, intended to fulfill specific software engineering needs. Our approach to classification is to measure the software system and build multivariate stochastic models for predictiing high risk system components. We present experimental results obtained by classifying Ada components into two classes: is or is not likely to generate faults during system and acceptance test. Also, we evaluate the accuracy of the model and the insights it provides into the error making process.
\end{abstract}

Key words: Optimized Set Reduction, data analysis, fault-prone Ada components, stochastic modeling, machine leaming, classification trees, logistic regression.

\section{Introduction}

It has been noted that a small number of software components are responsible for a disproportionately large number of faults in any large-scale system [BP84, SP88, MK92]. Therefore, if we can identify components that are likely to produce a large number of faults, we can concentrate the verification and testing processes on them. This allows us to optimize the reliability of our software system with minimum cost. To do this, we build quantitative models that predict which components are likely to contain the highest concentration of faults. However, building such models is a difficult task: it is often the case in software engineering that the data which is collected is minimal, incomplete and heterogeneous [BBT92]. This presents several problems for model construction and interpretation (e.g., small data sets, inaccurate models, outliers). Therefore, we need a modeling process that is robust to these problems, allows for the reliable classification of high risk components (those that have a high probability of generating a fault during system or acceptance test), and aids in the understanding of the causes of this high risk. This understanding is important because it can give us insight into the software development process, allowing us to take remedial actions and make better process decisions in the future.

In this context, we will examine the use of the following modeling approaches:

- Logisic regression, which is one of the most commonly used classification techniques [Agr90, HL89]. This technique has been applied to software engineering modeling [MK92], as well as other experimental fields, and will therefore be used as a baseline for comparison in this paper.

1 Research for this study was suppored in part by NASA grant NSG 5123 and NSF grant $01-5-24845$

$10014023 \mathrm{~L}$ 
Many assumptions and constraints inherent to this technique make it difficult to apply in a software engineering context: (1) non-monotonicity of the probability density function on the explanatory variable range (2) interactions between explanatory variables are difficult to take into account when performing exploratory data analysis with numerous explanatory variables.

- Classification trees, which are described in [BF+84]. They were used to address software engineering modeling issues in [PA+82, SP88]. A review may be found in [CE87, BBT92]. Their strengths stem from their simplicity and readability. Their weaknesses come from a lack of ability to extract and use all statistically significant trends and a tendency to include non-relevant and non-significant information in the tree.

- Optimized Set Reduction (OSR), which has been developed at the University of Maryland [BBT92] in the framework of the TAME project [BR88] and has already been applied to several software engineering applications [BBT92, BBH92, BTH93]. It is partially based on both machine leaming principles [Q86, BF+84], and univariate statistics [Cap88]. Our motivation for developing OSR, and a tool to support it, was to design a data analysis approach that matches, to the extent possible, the specific needs of multivariate empirical modeling for software engineering [BBT92]. OSR generates logical expressions which represent patterns in a data set. For instance, consider the following example of a simple pattem (logical expression) related to high fault concentration:

\section{Example 1:}

A compilation unit that imports numerous declarations from outside the subsystem in which it is developed, that shows a large average statement nesting level and an intense use of global variables is likely to generate fault reports during system and acceptance testing. The corresponding logical expression characterizing this class of compilation units would be: NONLOC_IMP $=$ High $\wedge(N E S T I N G=$ High $\wedge$ GLOBALS $=$ High $)$

In this paper, we intend to show that OSR may be used as an alternative to logistic regression or classification trees to generate empirical models of risk within a software system, and that it can yield more accurate results. We will discuss issues related to the interpretation of the generated models. In particular, we will demonstrate how OSR can be useful in (1) identifying characteristics of high-risk components in a large Ada system and (2) providing some understanding about how faults originate during the software development process

In Section 2, we present an evolved version of the OSR algorithm (an earlier version of the OSR approach was applied to project cost estimation and published in [BBT92]) which is intended to make OSR models more accurate and easier to interpret. Specifically, the new algorithm inproves the interpretability and the accuracy of the models in three ways. First, it provides a mechanism for dealing with the discretization of the explanatory variable ranges in an automated way. This better supports the requirement that our models need to be able to handle the problem of heteroscedascity (see R5 in [BBT92]) Secondly, we provide OSR with the ability to work with conjunctive predicates (which will be called predicates in this paper), allowing our models to elicit the effects of combinations of variables which were not visible in the previous version of OSR. Finally, we provide support for recognizing similarities among patterns, which aids the user in model interpretation. These second and third adaptations help OSR deal with the requirement that our models are able to handle interdependencies and interactions among the explanatory variables (see R4 in [BBT92]).

Also in contrast to [BBT92], this paper applies the OSR modeling technique to the issue of classifying Ada components as either low or high risk, as opposed to project cost estimation (prediction on a continuous range). Accordingly, we use logistic regression and classification trees as a baseline for evaluating the OSR results. (Preliminary and partial results of this research were presented in [BBH92] based on the analysis of FORTRAN systems). 
In Section 3, we present a validation of the OSR process, which is based on constructing models using data from a large Ada system developed at the NASA Goddard Space Flight Center. In Section 3.2 we compare the generated OSR models to both logistic regression and classification tree models with respect to their accuracy. In Section 3.3, we discuss the interpretability of the OSR models. Finally, in Section 4 , we outline the main conclusions of this paper and define the future directions of the research.

\section{Optimized Set Reduction}

Assume we want to assess a characteristic of an object. We will refer to this characteristic as the dependent variable (Y). The object is represented by a set of explanatory (known or assessable) variables (called Xs). These variables can be either discrete or continuous. Also, assume we have a historical data set containing a set of experiences that contain the previously cited Xs plus an associated actual $Y$ value. Our goal will be to determine which subset of experiences from the historical data set provides the best characterizations of the current object to be assessed.

Example 2: Assess the expected frequency of faults $(Y)$ that will be detected during system and acceptance test within a particular compilation unit. For instance, the Xs may be: complexity metrics, system architecture metrics or developer related evaluation of skills.

\subsection{The OSR Process}

First, we will introduce new terminology in an attempt to both formalize the intuitive concepts related to empirical modeling and give those concepts a furm grounding in the OSR context. Subsection 2.1.1 presents the notions informally to provide the reader with some intuition about the method. The rest of Section 2 will be more structured and formal in order to define more complex notions without ambiguity. Whenever needed, definitions will be formal specifications whereas others will be in algorithmic form.

\subsubsection{Basic Definitions}

Assume we have a historical data set consisting of $n$ experiences, where each experience consists of a value for a single dependent variable $(Y)$ and a set of values corresponding to a set of $m$ explanatory variables $\left(E V=\left\{X_{1}, X_{2}, \ldots, X_{m}\right\}\right)$. We define the term pattern vector to mean one of these such experiences. Assume the dependent variable's value domain (dom(Y)) is divided into a set of disjoint and exhaustive classes which can be either intervals (if the $Y$ is continuous) or categories (if the $Y$ is discrete). Each explanatory variable has its own value domain $\left(\operatorname{dom}\left(X_{i}\right)\right)$ which, like dom $(Y)$ is divided into a set $C$ of disjoint and exhaustive value classes $C=\left\{C_{\text {lass }} 1\right.$, Classi2... Classik $\}$. We define a measurement vector to be a pattern vector without the dependent variable $Y$. (Note that a measurement vector can be used to represent an object whose dependent variable value is not known, but is of interest

and which we wish to assess). The measurement vector value domain is $M V=\underset{i \in(1 . . m)}{\times} \operatorname{dom}\left(X_{i}\right)$. Likewise, the pattern vector value domain (i.e., the domain of the vectors in the data set) can be represented as $\mathrm{PV}=\operatorname{dom}(\mathrm{Y}) \times \mathrm{MV}$. We define PVS $\subseteq \mathrm{PV}$ to be a pattern vector set, representing the
historical data set.

Example 3: Suppose (Size $=100 \mathrm{LOC's,} \mathrm{Function} \mathrm{type}=$ computation) is a measurement vector characterizing a compilation unit Assuming $Y$ is \#faults, (\#faults $=6$, Size = $100 \mathrm{LOC}$ 's, Function_type $=$ computation) is a pattern vector characterizing a particular testing experience on a compilation unit. 
At the very heart of the OSR process, is what we call a singleton predicate. We define a singleton predicate to be a pair with the following form: $\left(X_{i}\right.$, Class $\left.i j\right)$ meaning that explanatory variable $X_{i}$ has a value belonging to Class $_{i j} \subseteq \operatorname{dom}\left(X_{i}\right)$. A singleton predicate (also written $X_{i} \in$ Class $i j$ ) is said to be TRUE for a measurement vector if that vector's explanatory variable $X_{i}$ value is an element of Class $i j$, otherwise, the singleton predicate is said to be FALSE for that vector.

Example 4: Size $\in[50,200)$ is a singleton predicate

Now that we have defined the notion of a singleton predicate, we can define other elements of OSR which are built upon this notion. For instance, we can define a conjunctive predicate (denoted Pred and simply called a predicate from here on) as the conjunction of singleton predicates. We will consider a predicate to be a set of singleton predicates, where the conjunction is implicit. A predicate is said to be TRUE for a given measurement vector if each of its constituent singleton predicates is TRUE for that vector. (Note that by defining a predicate to be a set (conjunction) of singleton predicates gives OSR the ability to elicit some of the complex interdependencies that exist between the explanatory variables, see requirement $\mathrm{R} 4$ in [BBT92]).

Example 5: Size $\in[50,200) \wedge$ Function_type $\in\{$ computation $\}$ is a predicate

A predicate may be used to characterize sets of pattern vectors. For example, if we define IS TRUE(Pred, pv) to yield TRUE if Pred is a true logical expression for the pattern vector $p v$, (i.e., each singleton predicate in Pred is true for $p v$ ), then we can define a predicate Pred and a subset PSS of the historical data set (PVS) such that IS_TRUE(Pred, pv) yields TRUE for each $p v$ in PSS. Similarly, we define SUBSET(PSS, Pred) to denote a subset of PSS characterized by Pred. Also, we define PSS to be equivalent to SUBSET(PSS, TRUE). Finally, MEMBER(X, Pred) yields the value TRUE if the variable $\mathrm{X}$ appears anywhere in Pred, FALSE otherwise.

\subsubsection{Optimal Subsets of Experiences}

In this section, we rigorously define the notion of "optimal subset of experiences" by defining the function OPT that extracts these subsets from a given historical data set. We will see in the next section that OPT is not directly implementable. Nonetheless, this definition should help the reader understand our goals at a first glance. These definitions, by their very nature are somewhat terse. However, the accompanying explanations should help the reader get an intuitive understanding of the process.

\section{- Definition 1: Normalized Entropy H(PSS, Y)}

This is the information theory definition of entropy that characterizes distributions, normalized to yield a value between 0 and 1 . This concept is commonly used in machine learning[M83] in order to assess the level of information provided by a distribution on a continuous or discrete range. It yields a value 0 when unambiguous information is provided and 1 when no information is provided.

$$
H(P S S, Y)=-\sum_{C l a s s Y, C C} p(P S S, \text { Class } Y j) \log _{1,1} p(P S S, C l a s s Y j)
$$

where,

. PSS is a set of pattern vectors

. Class $Y_{j}$ is a class defined on dom(Y)

- $\mathrm{p}\left(\mathrm{PSS}, \mathrm{Y}_{j}\right)$ is the prior probability that a vector which is an element of PSS has a dependent variable value belonging to the dependent variable class $Y_{j}$ 
- Definition 2: DIFFDIST(PSS $\left.{ }_{\mathrm{j}}, \mathrm{PSS}_{\mathrm{j}}, \mathrm{Y}\right)$

DIFFDIST(PSS, PSS $\left._{j}, Y\right)=$ TRUE if the two sets of pattern vectors characterized by PSS $_{i}$ and PSS show a statistically significant DIFFerence in DISTribution on the dependent variable ( $Y$ ) range and is FALSE otherwise. This function is based on binomial tests for proportions and is better described in [BBT92]. The statistical level of significance used as a threshold between TRUE and FALSE is subjective and is therefore defined by the user (e.g., 0.05, 0.1).

- Definition 3: VALID(PSS, mv)

This function yields TRUE if at least one predicate is TRUE for all the pattern vectors in PSS and for the measurement vector $m v$.

PSS ᄃPVS $\wedge$ mv $\in$ MV $\wedge \exists$ Pred such that $(\forall p v \in$ PSS , IS_TRUE(Pred , pv $) \wedge$ IS_TRUE(Pred, mv) ) $\Rightarrow$ VALID(PSS, mv)

- Definition 4: EMIN(PSS, PSS $\left.{ }_{\mathrm{j}}, \mathrm{Y}\right)$

EMIN(PSS, PSS,$Y$ ) $=$ TRUE if PSS $S_{j}$, a subset of PSS, shows a significantly different distribution from PSS on the $Y$ range (based on a predefined level of significance and according the result of the function DIFFDIST) and for all other subsets PSS $\mathrm{k}$ of PSS showing a statistically significant $Y$ distribution, $\mathrm{H}\left(\mathrm{PSS}_{\mathrm{j}}, \mathrm{Y}\right) \leq \mathrm{H}\left(\mathrm{PSS}_{\mathrm{k}}, \mathrm{Y}\right)$. EMIN stands for: Entropy is MINimum. In other words, EMIN tells us if PSS ${ }_{j}$ characterizes a subset with minimal possible entropy and that this low entropy is not likely to be due to chance.

$P S S \subset P V S \wedge P S S_{j} \subset P S S \wedge\left(D I F F D I S T\left(P S S_{j}, P S S, Y\right) \wedge\left(\forall P S S_{k} \subset P S S, k \neq j\right.\right.$, DIFFDIST(PSS $k$, PSS, Y) $\left.\left.\wedge \mathrm{H}\left(P S S_{j}, Y\right) \leq \mathrm{H}\left(P S S_{k}, Y\right)\right)\right) \Rightarrow$ EMIN(PSS, PSS, Y $)$

- Definition 5: OPT(PVS, mv, Y)

OPT yields a set of OPTimal subsets of pattern vectors of PVS (the historical data set) based on the definitions presented above. These subsets are characterized by predicates which are built based upon known information (i.e., $m v$ ) and show a minimal entropy. They can therefore be used for predicting the value of $Y$ with respect to $m v$.

Example 6: In Figure 1, based upon a given measurement vector $(m v)$ and a given historical dataset, the optimal subset extracted by OPT and characterized by the predicate on the left hand side of Figure 1 indicates a strong probability for $Y$ to lie in the interval $Y 2$. This may be used for predicting the class where the object described by $m v$ is likely to lie. Also, if $Y$ is defined on a continuous scale, the optimal subset expected value may be used as a prediction. 


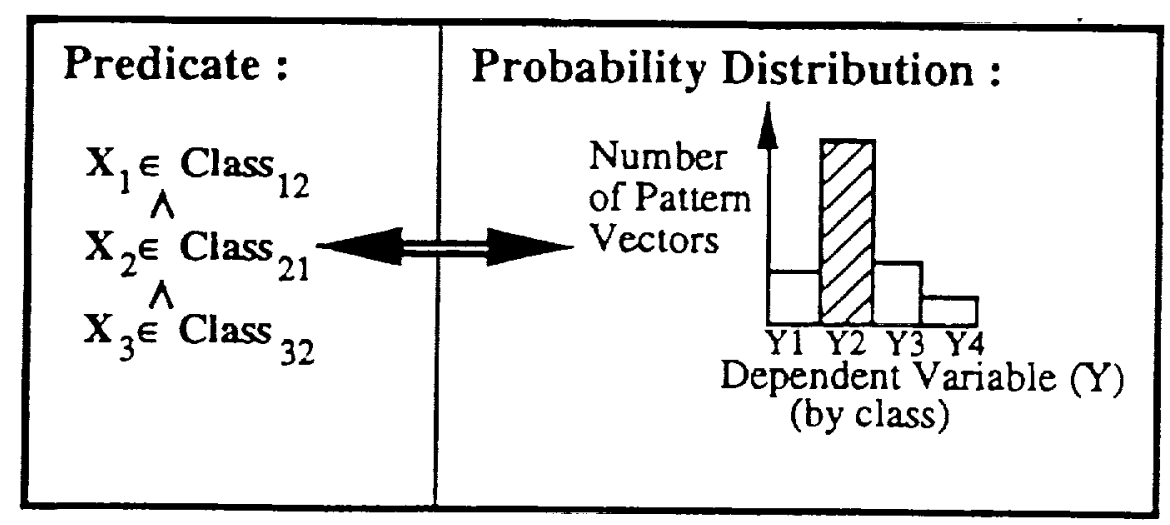

Figure 1: Classification with Extracted Subsets

Based on the primitives defined above, OPT may be defined as follows:

$$
\text { OPT (PVS, } m v, Y)=\{P S S \mid P S S \subseteq P V S \wedge \text { VALID }(P S S, m v) \wedge \text { EMIN(PSS, PVS, Y) }\}
$$

The function OPT as defined above defines optimal subsets of experiences with minimal entropies and characterized by optimal predicates. However, this is just a first step in the definition of an optimal search algorithm to extract datasets' patterns since there are several reasons why this simple function is not fully adequate to build empirical models to fulfill our needs. Some of these reasons are simply computational in nature while others are related to the loss of useful information.

-1: The number of possible singleton predicate combinations makes the execution time of the search of optimal predicates prohibitive without a search strategy.

-2: We are not only interested in the optimal subsets extracted by OPT but also by the predicates that characterize them. We want each generated predicate to contain only singleton predicates that have a significant impact on the resulting distribution entropy (see Figure 1). Thus, we can minimize the information necessary to identify optimal subsets and make the predicates more interpretable.

- 3: We need to extract information about the relative impact of the various singleton predicates within the optimal predicates.

- 4: The conditions under which singleton predicates or predicates appear relevant have to be determined.

Therefore, we will now define an algorithm which addresses these issues, discussing its relationship to the function OPT. This is the Optimized Set Reduction process which can roughly be described by a three step recursive algorithm where entropy is optimized in a stepwise manner.

\subsection{The OSR Algorithm}

The goal of the OSR algorithm is to produce a set of patterns which characterize the trends observable in the historical data set while addressing the four modeling issues mentioned above. In this context, the notion of pattern is based upon the notion of predicate as defined above while addressing some of the mentioned modeling needs. This definition of pattern intends to be both useful for predicting and suitable to interpretation. 
In subsection 2.2.2, we shall describe the OSR algorithm in detail. However, before doing so, we need to define a number of preliminary concepts that are used in the algorithm.

\subsubsection{Preliminary Definitions}

\section{- Definition 6: OSR Pattern}

As mentioned above, OSR generates patterns. A pattern is an ordered conjunction of predicates which characterizes a subset of PVS that shows a minimal entropy distribution. The notion of ordering will be represented by the "ORDERED AND" symbol $(\stackrel{\wedge}{\rightarrow})$. It is logically equivalent to the symbol $(\wedge)$ with the exception that predicates to the right of $a \wedge$ symbol are relevant only when all predicates to the left of the symbol are already TRUE. The notion of order is introduced here to capture information about the conditions under which a predicate is relevant and does not have any logical impact on the characterization of optimal subsets We will call the ordered expression to the left of a given predicate in a pattern the context of the predicate. This addresses issue number 4 mentioned above.

Example 7: Define two predicates

\section{Pred $1=$ SUBSYSTEM $\in$ REAL $T$ TME CONTROL $\wedge$ SUBSYSTEM $\in$ LARGE Pred $2=$ \#GLOBAL VARIABLES $\in$ LARGE}

If we assume the pattern $\operatorname{Pred}_{1} \wedge \operatorname{Pred}_{2}$ was generated by OSR, we can see that this pattern characterizes a pattern vector set suggesting a high risk which is defined, in this particular example, as the probability of detecting errors that are difficult to correct during the test phases (see Figure 2).

This pattern (Pred $1 \wedge$ Pred $_{2}$ ) has a specific interpretation associated with it. Pred $I$ is a nonsingleton predicate and Pred 2 is relevant within the context of Pred 1 . This pattern implies the following interpretation. If a subsystem is both large and real time, then it is significantly more likely to be of high risk than a random subsystem. However, it does NOT suggest that either real time subsystems or large subsystems independently increase the probability that a subsystem will be of high risk. Also, within the context of large, real time subsystems, subsystems with a large number of global variables have a significantly greater probability of being high risk than those with a small number of global variables. However, this pattem does NOT suggest that a large number of global variables has a significant impact on the probability that a subsystem will be of high risk outside the context of large, real time subsystems. (More details concerning pattern generation and interpretation will be presented later in the paper.) 


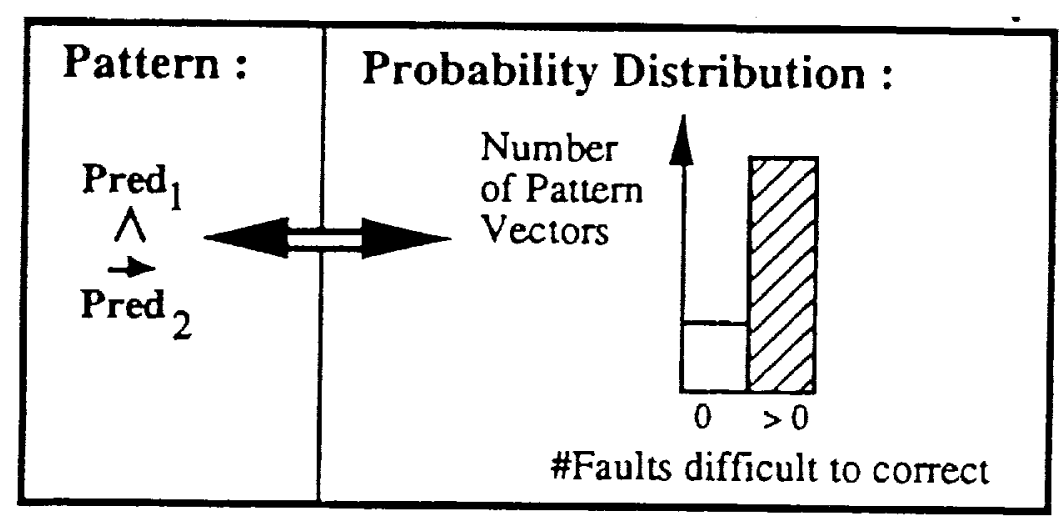

Figure 2: Classification Using Patterns

\section{- Definition 7: DISCRETIZE(PSS, $\mathrm{X}_{\mathrm{i}}$ )}

Given a particular subset of pattern vectors (PSS), we want to divide/cluster the ranges/categories of the explanatory variables into an exhaustive and disjoint set of classes (Classil ... Classik for the explanatory variable $\mathrm{X}_{\mathrm{i}}$ ) based on a meaningful class creation techniques. This is used to both define singleton predicates and to better satisfy the problem of heteroscedascity, i.e., requirement R5 of [BBT92] which states that an explanatory variable may be a good predictor on a part of its range/value domain while a mediocre predictor otherwise. Clustering of discrete categories can only be performed by the user by defining taxonomies. Numerous techniques are available in the literature to create intervals on continuous / ordinal ranges (e.g., cluster analysis) [DG84]. However, none appear to have satisfactory properties for our problem. Therefore, classes are created for continuous / ordinal explanatory variables according to the procedure DISCRETTZE briefly presented below and described in Appendix II.

DISCRETIZE(PSS, $\mathrm{X}_{\mathrm{i}}$ ) defines classes on the range of $\mathrm{X}_{\mathrm{i}}$ (a particular continuous or ordinal explanatory variable) based on a pattern vector subset PSS. This algorithm has the following properties:

- Either all or some of the classes should show distributions on the $\mathrm{Y}$ range that are significantly different than the distribution resulting from the union of those classes. If not, differentiating these classes and creating new pattern vector subsets is meaningless.

- The algorithm handles monotonic and non-monotonic underlying distributions on the $\mathrm{Y}$ range.

-The algorithm is not oversensitive to the addition or deletion of few pattern vectors so stable patterns are generated.

Our goal is to take into account the above constraints and to minimize the average entropy across the created classes in order to have classes as homogeneous as possible with respect to the dependent variable values of their pattem vectors. Figure 3 illustrates the output of the algorithm. We assume an actual underlying and unknown non-monotonic probability density function and an observed sequence of $Y$ values on the explanatory variable $X$ range. We also assume two classes $(1,2)$ are defined on the $Y$ value domain. Using the DISCRETIZE algorithm produces Boundaryl and Boundary 2 in Figure 3, which creates the corresponding set of three explanatory variable value classes across the $\mathrm{X}$ range. 


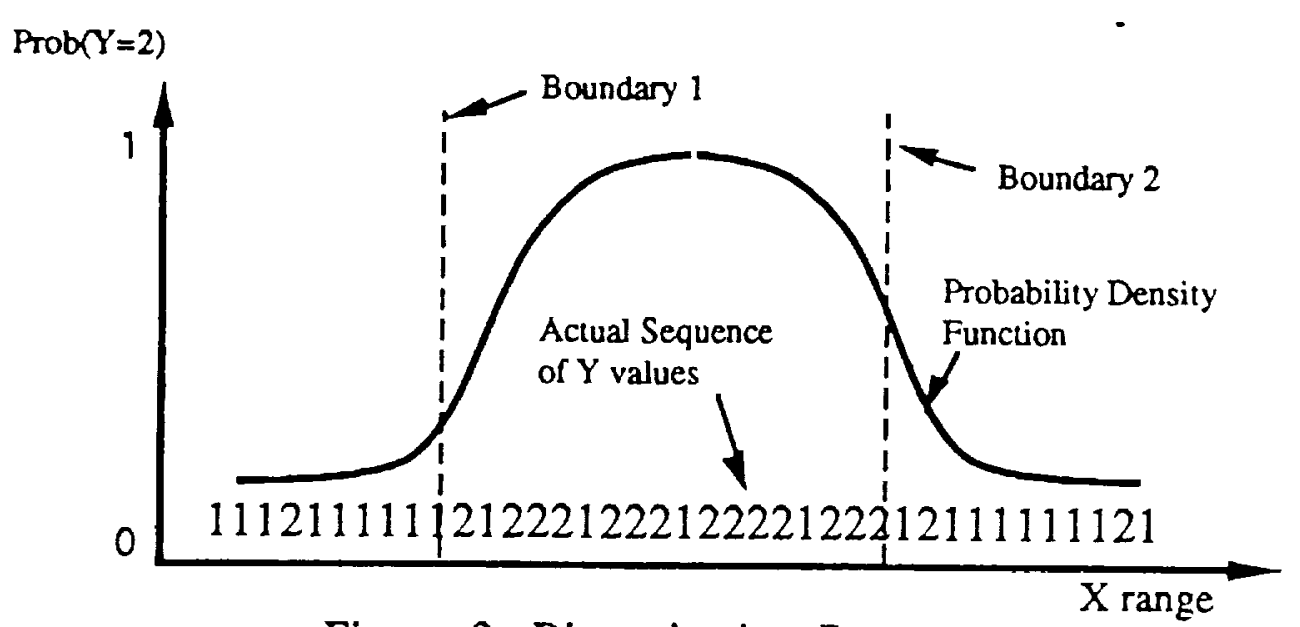

Figure 3: Discretization Process

\section{- Definition 8: GENERATE_SINGLETONS(PSS, mv)}

Let PSS represent the considered pattern vector set and let $m v$ be a measurement vector. The classes defined by DISCRETIZE for each explanatory variable $\mathrm{X}_{\mathrm{i}}$ give us a set of singleton predicates: $\left\{\mathrm{X}_{\mathrm{i}} \in\right.$ Classil $1, \ldots, X_{i} \in$ Classik). GENERATE_SINGLETONS(PSS, mv) generates the set of all singleton predicates SP such that $S P=\left\{\operatorname{Pred}_{i} \mid\right.$ IS_TRUE $\left.\left(\operatorname{Pred}_{i}, m_{v}\right)\right\}$.

- Definition 9: SIG_PREDICATE(PSS, Pred, Y)

The predicate Pred is said to be significant for the data set PSS if SUBSET(PSS, Pred) shows an entropy lower than the one of PSS and if their distributions on the $Y$ range show statistically significant differences.

PSS $\subseteq$ PVS $\wedge($ H(SUBSET(PSS, Pred), Y $)<H(P S S, Y) \wedge$

DIFFDIST(PSS, SUBSET(PSS, Pred), Y)) $\Rightarrow$ SIG_PREDICATE(PSS, Pred, Y)

Example 8: Assuming two dependent variable classes ([low, high]), suppose Pred characterizes a subset whose distribution across the two classes is $[10,7]$. This subset shows an entropy which is lower than the entropy of PSS, which had a distribution [100,75], but the difference is not statistically significant since the proportion of pattern vectors in each class is practically the same. A binomial test for proportions [Cap88] is used to assess the significance of the observed difference in entropy.

- Definition 10: MINIMAL(PSS, Pred, Y)

The predicate Pred ${ }_{\mathrm{i}}$ is said to be minimal for the pattern vector set PSS if it characterizes a subset of PSS which shows a significantly different distribution across the $Y$ classes and there exists no other predicate Pred $_{j} \Rightarrow$ Pred $_{i}$ such that Pred $_{j}$ characterizes a subset of PSS which shows a significantly different distribution across the $Y$ classes. Otherwise, Pred $_{i}$ contains more singleton predicates than is necessary to significantly improve the entropy and is not considered to be minimal.

SIG_PREDICATE(PSS, Pred,$Y) \wedge\left(\forall j\right.$, Predj $_{j} \Rightarrow$ Pred $_{\mathrm{i}}, \mathrm{j} \neq \mathrm{i},(\neg$ SIG_PREDICATE(PSS,Pred, $\left.\mathrm{Y})\right)$ $\Rightarrow$ MINTMAL(PSS, Pred, Y ) 
Example 9: Assume that the predicate Pred $_{l}=$ SUBSYSTEM $\in$ REAL-TIME CONTROL $\wedge$ SUBSYSTEM $E$ LARGE yields, in a defined context, an entropy of 0.5 (assumed to yield a significanlly different distribution from the parent set). If Pred $2=$ SUBSYSTEM $\in$ REALTIME CONTROL by itself yields an entropy of 0.5, Pred $_{l}$ is not minimal.

- Definition 11: VALID_PREDICATES(PSS, PRED $, S P, Y$ )

Let PSS represent a set of pattern vectors and $\mathrm{PRED}_{\mathrm{c}}$ be a set of predicates which define the context characterizing PSS. Let SP be a set of singleton predicates and $Y$ be the dependent variable.

Assuming that the set SP has been created by using GENERATE_SINGLETONS, we generate the set of all predicates which are conjuncts of the singletons in SP and which are minimal with respect to PSS (as defined above), as long as they do not use any explanatory variable $\mathrm{X}$ that appears in $\mathrm{PRED}_{\mathrm{c}}$. These predicates are called valid and are the ones that appear potentially useful for extracting subsets of PSS with high predictive power for $m v$ on the $Y$ range. With respect to the implementation of this procedure, the user may restrict the search space by fixing a maximum number of singleton predicates per predicate. However, some complex but meaningful predicates may not be extracted by doing so.
VALID_PREDICATES(PSS, PRED, $, S P, Y)=\left\{\operatorname{Pred}_{\mathrm{i}} \mid\right.$ MINIMAL(PSS, Pred $\left._{\mathrm{i}}, \mathrm{Y}\right) \wedge \operatorname{Pred}_{\mathrm{i}} \subseteq \mathrm{SP} \wedge$ $\left(\forall \mathrm{j}\right.$, Pred $_{\mathrm{j}} \in \operatorname{PRED}_{\mathrm{c}}, \forall \mathrm{X}$ such that $\mathrm{X} \in\left\{\mathrm{X}_{\mathrm{k}} \mid \mathrm{X}_{\mathrm{k}} \in \mathrm{EV} \wedge \operatorname{MEMBER}\left(\mathrm{X}_{\mathrm{k}}, \operatorname{Pred}_{\mathrm{j}}\right)\right\}$, $\neg$ MEMBER $\left(X\right.$, Pred $\left.\left._{\mathrm{i}}\right)\right\}$

- Definition 12: EXTRACT_SUBSETS (PSS, PRED)

Let PRED be a set of predicates. A set of subsets, where each subset is characterized by one and only one predicate in the set PRED, is extracted from PSS.

EXTRACT_SUBSETS (PSS, PRED) $=\left\{\right.$ PSS $_{\mathrm{i}} \mid$ Pred $_{\mathrm{i}} \in$ PRED $\wedge$ PSS $\mathrm{i}_{\mathrm{i}}=$ SUBSET $\left(\right.$ PSS, Pred $\left.\left._{\mathrm{i}}\right)\right\}$

\subsubsection{The Algorithm}

When the dependent variable's value domain is defined on a continuous scale, its range is assumed to be divided into intervals / classes. These classes are fixed and will be used throughout the algorithm. These intervals are usually defined according to two main criteria: the size of the dataset and the specific use of the model. The larger the data set, the narrower the classes may be so that the model can produce a more accurate response. Also, the definition of these classes must also take into account the future use of the model, e.g., they represent clusters on the $Y$ range or a finite number of situations suggesting alternative actions.

\section{Example 10:}

Assume that the range of the dependent variable $(Y)$ is an integer range from 0 to 5 , indicating the number of fault reports that were generated for a component during system and acceptance test. Then, we may decide to define the following dependent variable classes:

$$
\begin{aligned}
& \text { Class } Y 1=Y \text { in }[0,1) \quad \text { Low Risk Components } \\
& \text { Class } Y 2=Y \text { in }[1,+\infty) \text { High Risk Components }
\end{aligned}
$$

Let PSS be a set of pattern vectors, let $m v$ be a measurement vector characterizing the object to be 
classified on the $Y$ range, and let $\mathrm{PRED}_{\mathrm{c}}$ be the set of predicates composing the pattern characterizing the set PSS. Recall that we cannot use OPT directly. However, OSR(PSS, mv, PRED,$Y$ ) heuristically returns a set of "optimal" subsets using the algorithm defined below.

OSR(PSS, mv, PRED P, $\mathrm{Y})$

- Step 1: SP = GENERATE_SINGLETONS (PSS, mv)

* Generate a set of optimal singleton predicates based on the pattern vector set PSS */

$l^{*}$ and for the measurement vector $m v$

- Step 2: PRED = VALID_PREDICATES (PSS, PRED $C, S P, Y$ )

$1^{*}$ Generate all the valid predicates based on the available set of singleton predicates

/* SP, the current context defined by $P R E D_{C}$, and its corresponding pattern vector set PSS. */

- Step 3:

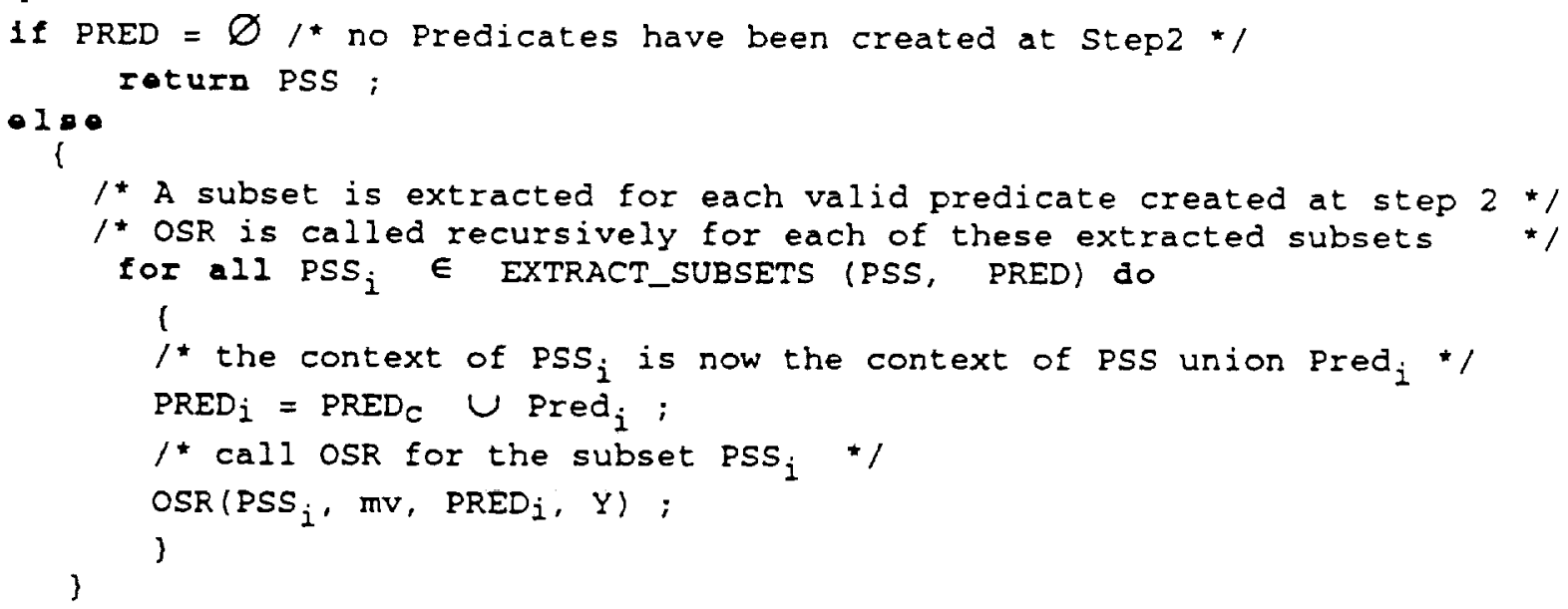

Initially, call OSR(PVS, $\mathrm{mv}, \varnothing, \mathrm{Y}$ ) where PVS is the historical data set.

The OSR algorithm can be viewed as a recursive function of OPT as described below. PVS is the historical data set and $m v$ the vector describing the object to be assessed. Let us assume we modify the definition of the function VALID, which is used to build OPT, so that the function MINTMAL is included in it. Then, VALID becomes the following:

PSS $\subset$ PVS $\wedge m v \in M V \wedge \exists$ Pred $_{j}$ such that $(\forall p v \in$ PSS , IS_TRUE(Pred, pv $) \wedge$ IS_TRUE(Pred ${ }_{i}$, mv) $\wedge$ MINIMAL(PSS, Pred $\left.\left._{i}, Y\right)\right) \Rightarrow$ VALID(PSS, mv, Y)

Then, assuming the definition of OPT uses this new definition of VALID, we can then define OSR in the following way:

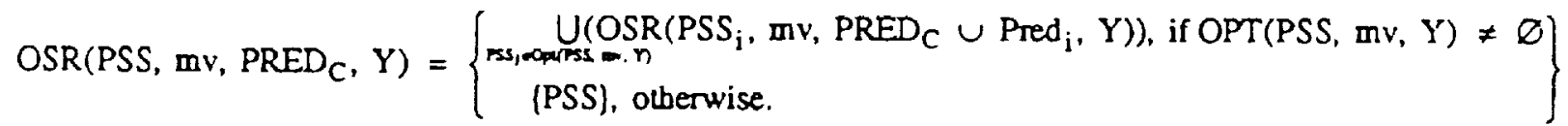

Note that at each level of recursion, a minimal subset of pattem vectors is extracted. These recursively nested, extracted subsets are each characterized by a predicate in a context. Thus, if we implicitly order the paths, the ordered conjunction of predicates along each recursive path is a pattem (see Definition 6 


\section{and Figure 4).}

The subsels of PVS extracted by OSR for a particular mv may be used for the classification of $Y$ for $m v$. Also, if patterns are extracted for each mv in PVS, the resulting set of pattems may be used for the interpretation of the impact of the explanatory variables on the dependent variable in a particular development environment. These issues will be addressed in the next sections.

\section{Example 11}

In figure 4, we can see how OSR patterns are generated during the subset extraction process. At the first (highest) level in the hierarchy, suppose $\angle$ NUM_IMPORTS = HIGH $>$ is a predicate which is minimal, causing the extraction of Subset 1 . At the second level, suppose the two place predicate $\angle$ NESTTNG $=\mathrm{HIGH} \wedge \mathrm{CMPLX}=\mathrm{HIGH}>$ was found to be minimal. Then, by tracing the hierarchy down this particular path, OSR generates the following pattern, which corresponds to the extracted subset 1.1:

\section{NUM_IMPORTS $=\mathrm{HIGH} \wedge($ NESTING=HIGH $\wedge$ CMPLX $=\mathrm{HIGH})$}

Also, each path in the hierarchy from the top set (PVS) to a bottom level subset is marked by its own pattern. Thus, OSR creates a set of patterns, (i.e. all the paths in the hierarchy).

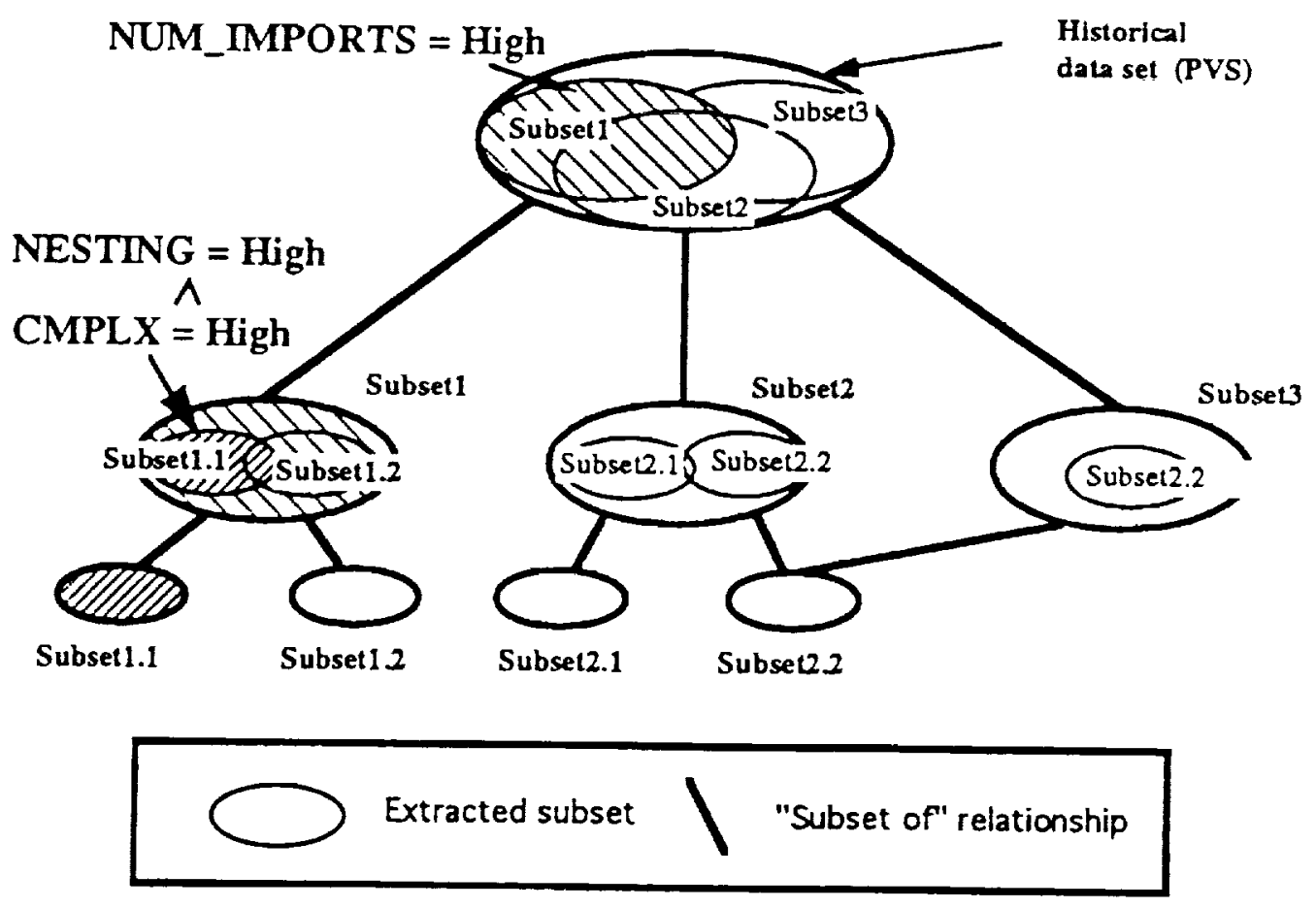

Figure 4: An Example of OSR Hierarchy

Each path of the hierarchy represents a path that the extraction process may have taken during OSR. Accordingly, each path is characterized by an ordered conjunction of predicates, i.e., a pattern. Each final extracted subset (i.e., leaves of the hierarchy) forms a probability distribution across the dependent variable range. This distribution is a valuable piece of information and can be used in several ways. For instance, if the dependent variable is discrete, the dependent variable class containing the largest number 
of pattern vectors may be selected as the most likely class for the new object's $Y$ value to lie in. Alternatively, we may consider using a Bayesian approach. That is, we could define a loss/risk function [BBT92] and select the dependent variable class yielding the minimum expected loss. Finally, note that several leaves may have distributions that yield contradictory or dissimilar trends. Therefore, several pattern classifications (i.e., hierarchy leaves) are used to make a final global classification based on predefined decision rules. In order to perform such decisions effectively, we need to be able to evaluate the accuracy of the identified patterns (e.g., hierarchy branches). This is the topic of the next subsection.

\subsection{Assessing the Accuracy of Patterns}

In order to generate patterns and assess their accuracy, we use OSR in the context of the technique called $V$-fold Cross Validation [BF+84]. For each pattern vector $p v$ in the historical data set, we can run the OSR algorithm using PVS - $\left(p v_{i}\right)$ as the initial data set and using the measurement vector composing $p v$ as $m v$. The pattern vector $p v$ is removed from the data set in order to avoid any bias in the results. Thus, each time we run OSR, we know the actual value of the dependent variable we are trying to classify. This allows us to not only extract specific patterns for each pattem vector in the data set, but we are also able to classify each generated pattern as right or wrong at the time it is generated. The set of patterns generated through this iterative process forms a representation of the trends observable on this particular data set which we will call a Specific Pattern Set (SPS).

The SPS may be viewed as a hierarchical model (see figure 4) of the historical data set. Many of the patterns in the SPS will be the same or similar and will therefore form classes of patterns. For each of these classes, based on the SPS, we can evaluate statistics such as pattern reliability (i.e., percentage of correct classification when the pattern is used) and pattern reliability significance (i.e., the probability that the observed reliability is greater than or equal to the one expected through a random classification by chance). These statistics can then be used to evaluate the pattem based predictions as explained in the subsequent paragraphs. Thus, even though incomplete / partial information is available in the historical data set, accurate patterns may still be generated in some cases.

Recall that we assumed the patterns generated by OSR have the following ordered conjunctive normal form:

$$
\text { Predicate } \stackrel{\wedge}{\rightarrow} \text { Predicate } 2 \stackrel{\wedge}{\rightarrow} \cdots \stackrel{\wedge}{\rightarrow} \text { PredicateN }
$$

Also, recall the order in which the predicates appear is relevant in order to determine the contexts where they are relevant. A predicate is relevant only when the conditions defined by its preceding / parent predicates (i.e., the context of a predicate) are true.

Let Class $Y_{i}$ be dependent variable class $i$. Let $T$ be the number of generated pattern instances Patter $m_{j}$ that predict Class $Y_{i}$. Let $C$ be the number of pattern instances which correctly predict Class $Y_{j}$ (based on the actual $Y$ value of the pattern vector for which the pattern was produced).

Then we define the reliability of Pattern $j$ with respect to the dependent variable class Class $Y_{j}$ as:

$$
\mathrm{R}\left[\text { Class } Y_{\mathrm{i}} ; \text { Patternj] }=\mathrm{C} / \mathrm{T}\right.
$$

The probability that a pattern appears $\mathrm{T}$ times yielding a particular classification $\mathrm{Class}_{\mathrm{i}} \mathrm{C}$ times correctly by chance $(\mathrm{P}(\mathrm{C}, \mathrm{T}, \mathrm{P}))$ can be expressed by the binomial distribution:

$$
P(C, T, p)=\frac{T !}{C !(T-C) !} P^{c}(1-p)^{T-c}
$$


where, $p=p\left(\right.$ Class $\left._{i}\right)$, i.e., the prior probability that the value of the dependent variable is in Class $Y_{i}$.

If the pattern reliability $R$ is equal to 1.0 , then the binomial equation can be simplified and the level of significance is simply $p^{\top}$. If $R$ is below one, then the pattem reliability significance $R S$ can be calculated using the following formula:

$$
R S=\sum_{j=0}^{T-C} P(C+j ; T ; p)
$$

Example 12: For a given pattern, suppose that:

$$
\begin{aligned}
& \mathrm{C}=10 \quad \text { (the number of times that the pattern was correct during the } \\
& \mathrm{V} \text {-fold Cross Validation) }
\end{aligned}
$$

Also, suppose that there are exactly two dependent variables classes and an uniform distribution in the historical data set, so that the prior probability of a pattem predicting each class is 0.5 for each dependent variable class.

Then, using the above formulas, this pattern has the following reliability and reliability significance.

$$
\begin{aligned}
& R=0.83 \\
& R S=0.019
\end{aligned}
$$

Since we are able to differentiate significantly reliable patterns from the non-significant and/or unreliable ones, we are able to know the reliability of a classification when we make it. That is, when we are trying to assess a new object, we run the OSR algorithm using that object as the measurement vector. This process extracts a set of patterns specific to that object. Then, when making a classification for this object, we know that a classification based on a reliable pattern with a sufficient level of significance (e.g., RS < 0.05) is believable, whereas, one based on a reliable pattem with a poor level of significance is not.

Thus, our decision process is based on the R's and RS's of each pattern in the hierarchy. Pattern reliability is used for classification while the variations in pattern entropy are used for interpretation. Although a reliable pattem always shows a low entropy, the opposite is not true (for reasons beyond the scope of this paper).

Note: a poor reliability means that a pattern is not robust to "noise" (i.e., the dependent variable variations created by non-measured phenomena). A poor reliability significance may mean that the pattern is a result of noise or more complex phenomena resulting from the OSR process (again beyond the scope of this paper.

\subsection{Support for Interpreting Patterns}

As we have seen patterns are useful for classifying variables of interest. However, more importantly, they are also useful in providing understandable $/$ interpretable models. Patterns are much easier to interpret than regression coefficients. First of all, OSR takes into account interactions between explanatory variables, i.e., the fact that an explanatory variable can have a strong impact in a certain context and not be relevant in another one. These interactions do not have to be known before building 
the model as opposed to interaction terms in logistic regression [HL89]. Secondly, as we will see, a process (described below) can be defined to show strong associations that exist in a given context (this is needed to satisfy R4 of [BBT92]). Finally, the variation in entropy generated by a paricular predicate can help assess the significance of the impact of an explanatory variable (on the dependent variable) within a certain context. However, interpreting the raw patlerns would force the user to deal with useless complexity. Many of these patterns are similar and should not be differentiated. This can prevent the user from getting a clear picture of the model trends. Therefore, the patterns generated by the OSR process need to be grouped in order to make them more easily understandable and interpretable. This can be done using a formally defined statistical process (described below) where the user fixes the desired level of "similarity" between pattern by assigning values to a small set of parameters.

Let us define two patterns PT1 and PT2:

$$
\begin{aligned}
& \text { PT1: Predi } \stackrel{\wedge}{\rightarrow} \text { Predj }_{j} \\
& \text { PT2: Pred } i \stackrel{\wedge}{\rightarrow} \text { Predk }
\end{aligned}
$$

Suppose in the context where Predi is true, the pattern vector subset for which Predj is true happens to show a strong association with the one for which Predk is true. This implies that these predicates capture basically the same phenomenon. The strength of the association can be assessed by using normalized Chi-squared based statistic such as Pearson's Phi [CA88]. A Chi-squared test can be performed to assess the statistical level of significance of such an association. The two patterns will be merged into one signifying that the selection of one predicate, or the other, during the OSR process, occurred randomly. This is a result of slight differences between the two predicates and therefore distinguishing between them does not help in the understanding of the object of study. This phenomenon is mainly due to complex interdependencies between $X s$ that are often underlying the software engineering data sets.

In order to decide whether or not two strongly associated predicates should not be differentiated, the user declares a Phi value which represents the minimal degree of association necessary to assume two predicates as similar. This process of merging patterns based on the similar predicates principle yields the resulting pattern PT $\{1,2\}$ which contains the composite predicate (Pred $v \vee$ Pred $k$ ) implicitly meaning that its two component predicates are interchangeable in this context.

$$
\operatorname{PT}\{1,2\}: \operatorname{Pred}_{\mathrm{i}} \stackrel{\wedge}{\rightarrow} \operatorname{Pred}_{\mathrm{j}} \vee \operatorname{Pred}_{\mathrm{k}}
$$

Let us define a composite predicate to simply be a disjunction of predicates.

Example11: Assume that in the context of a subsystem that has for focus data processing, most of the components with a large number of SLOCs are also the ones with a large Halstead's volume V. PT1 and PT2 will be merged if the level of association between the two second position predicates (who are in this case singletons) is higher than the "Phi" threshold defined by the user.

$$
\begin{aligned}
& \text { PT1: SUBSYSTEM } \in \text { REAL-TIME CONTROL } \stackrel{\wedge}{\rightarrow} \mathrm{V} \in \text { LARGE, } R=0.90, \mathrm{RS}=0.06 \\
& \text { PT2: SUBSYSTEM } \in \text { REAL-TTME CONTROL } \stackrel{\wedge}{\rightarrow} \text { SLOC } \in \text { LARGE, } R=0.92, \mathrm{RS}=0.07 \\
& \text { PT\{1,2\}: SUBSYSTEM } \in \text { REAL-TIME CONTROL } \stackrel{\wedge}{\rightarrow} \mathrm{V} \in \text { LARGE } \vee \text { SLOC } \in \text { LARGE) }
\end{aligned}
$$




$$
R=0.91, R S=0.01
$$

In this situation where PT1 and PT2 are both reliable but show a small number of occurrences in the specific pattern set (see previous section), then they will be associated with weak levels of significance (RS). Merging them will increase this level of significance and keep the reliability (R) constant if the used Phi threshold is high enough.

Automated merging of similar patterns can be performed if the user provides either a Phi value or a level of significance that corresponds to an unambiguous definition of pattern similarity.

In a similar manner, we can define a second merging principle. Suppose we have the same two patterns as defined above:

$$
\begin{aligned}
& \text { PT1: Predi } \stackrel{\wedge}{\rightarrow} \text { Pred }_{j} \\
& \text { PT2: Pred } i \stackrel{\wedge}{\leftrightarrow} \text { Predk }_{k}
\end{aligned}
$$

However, this time suppose that Pred $_{j}$ is the singleton predicate $\mathrm{X}_{1} \in$ Class $_{\mathrm{km}}$ and Pred $_{k}$ is the singleton predicate $\mathrm{X}_{1} \in$ Class $_{\mathrm{kn}}$ where Class $\mathrm{km}$ is a neighbor class of Class $\mathrm{kn}$ (their boundaries may overlap). In this particular case, if the two patterns characterize subsets with no statistically significant difference in distribution on the dependent variable range, then they can be merged. This is because the variation from one class to the other seems to have a non-relevant effect on the dependent variable under the context where Predi is true. Therefore, in order to assess if merging is possible, the probability that differences between distributions are random is calculated. For each dependent variable class, the proportions of pattern vectors are compared between the two distributions by calculating the probability that difference in proportion is due to randomness. If for all dependent variable classes, the resulting minimum probability is above a user-defined critical probability value, we accept the hypothesis that there is no significant difference between the two distributions. In the tool developed to support the OSR approach, this is calculated through a binomial test for proportions.

Example12: Assume that in the context of components with a large number of SLOCs and a large Halstead's volume $V$, the programmers experience of the programming language (ordinal factor on a scale 1-5) is a significant factor. Both PT1 and PT2 show a first position predicate which is the result of a previous merging according to the first principle presented above. Their second position predicate is similar but not identical. PT1 and PT2 will be merged into PT $\{1,2\}$ if the level of similarity between the two second position predicates (who are in this case singletons) is higher than the threshold defined by the user.

$$
\begin{aligned}
& \text { PT1: }(V \in L A R G E \vee S L O C \in \text { LARGE) } \stackrel{\wedge}{\rightarrow} \text { EXPERIENCE } \in[1,2) \\
& \text { PT2: }(V \in \text { LARGE } \vee S L O C \in \text { LARGE) } \stackrel{\wedge}{\rightarrow} \text { EXPERIENCE } \in[2,3) \\
& \text { PT }\{1,2\}:(V \in \text { LARGE } \vee S L O C \in \text { LARGE) } \stackrel{\wedge}{\rightarrow} \text { EXPERIENCE } \in[1,3)
\end{aligned}
$$

Both of the merging principles defined above can be used simultaneously in order to obtain more significant and interpretable patterns. However, the merging process using both of them must be carefully defined. We have built a prototype tool where such mechanisms have been completely automated. A more precise definition of the pattern merging algorithm is presented in Appendix II. 


\section{Validating the Approach}

In order to validate the OSR approach, we need to compare it to standard modeling processes that can be used for classification: logistic regression [HL 89], classification trees [S92].

Our definition of a high risk component (procedure or function) is: any software component where errors were detected during system and acceptance test. Low risk is used to identify the remaining components of the system. In particular, we wish to build models that identify high risk components for a particular category of errors: ones that characterize an incorrect reading or writing in a variable or a data structure.

\subsection{Data Description}

The data set was created using data collected from 146 components of a 260 KLOC Ada system. We selected randomly an equal number of both low and high risk components in the used data set. This was done in order to construct unbiased classification models. We selected all the high risk components identified during test phases and we randomly introduced an equivalent number of low risk components among those available. A larger number of low risk components in the data would lead all modeling techniques to generate models more accurate for the low risk class and would therefore provide mediocre models for the high risk class (i.e., their results would not be representative of the actual capability of the models in terms of accurately identifying high risk components).

The explanatory variables used to construct the models are static code and design metrics. Some of these metrics are taken from a project whose goals were to build multi-variate models of software quality based on architectural characteristics of Ada designs [AES90,AE92,AE+92]. Others are well known component level complexity and size measures[BP84]. We will first summarize the architectural approach to measurement taken in this project and then define the assumptions upon which the analysis was conducted.

The architectural view of the Ada system can be derived by identifying the major components of the system, and determining the relationships among them. The library unit aggregation (LUA), or the library unit and all its descendant secondary units [AES90], provides an interesting concept for an Ada system. Relationships between LUAs can include the importing relationship, or the relationship between an instantiation and its generic template. The increased use of Ada as a design as well as implementation language provides an opportunity to better assess the final product in its intermediate stages. Since the design and the final product are written in the same language we can use tools developed for analysis of Ada source code to provide an automated means for analyzing Ada designs. This automation is essential if one is to frequently measure and assess the design.

The metrics used in this study are derived from the architecture of the system, and were obtained by an automated static analysis of the source code using the ASAP static analysis program [Dou87], UNDX utilities, and the SAS statistical analysis system. At the hear of the measures are counts of declarations in an LUA - whether they are declarations made in the LUA, declarations imported to the LUA (i.e., declarations made in another LUA made visible by a "with" clause), declarations exported by the LUA (i.e., declarations made in the library unit, and visible to other units that import the LUA), or declarations hidden from these imporing units (i.e., declarations made in the body and subunits).

The collection of metrics were developed from hypotheses about the nature of the software design process and further details can be found in [AES90,AE92,AE+92]. These, in addition to other raw measures extracted from the source code were used in this study. The metrics include ratios designed to indicate the extent of context coupling, visibility control, locality of imports, and parametrization. These characteristics are based on the following underlying assumptions: 
- Assumption 1 (Context coupling): Importing and/or exporting large amount of declarations may require complex interfacing with the other LUA's of the system and is expected to be an errorprone factor.

- Assumption 2 (Parametrization): The average number of parameters per program unit declaration in the LUA should have an impact on the probability of generating defects. The larger the parametrization of the LUA, the larger the number of abstractions to be dealt with, the greater the difficultly for a designer or a programmer to keep in memory their respective role, the more complex it becomes to handle interaction with others LUA's.

- Assumption 3 (Visibility control): The ratio of cascaded impors (declaration imports to a unit and whose visibility cascades to it's descendent units $[A E+92])$ to direct imports in the LUA. This concept captures the extent to which declarations are imported to where they are needed in the LUA. The larger the number of visible declarations unrelated to the problem addressed at a particular location in the LUA, the larger the risk of confusion or misunderstanding of those program abstractions.

- Assumption 4 (Reuse): A high ratio of reused code in a LUA denotes the familiarity / understanding with the problem addressed and the computer-based solution, i.e., the LUA interface with other LUA's, its component interfaces and its data structures. This is expected to lower the probability of defect.

In addition to the architectural metrics mentioned above, two main categories of component complexity metrics may be identified as well: size of the component and the structural or control flow complexity of the component.

- Assumption 5 (component size): Different measures of size were used: the total number of Ada statements, the number of executable Ada statements and the number of source lines of code. Size measures have shown in the literature to be related to the probability of generating defects [SP88, MK92].

- Assumption 6 (structural complexity): The structural complexity of the code should affect the probability of generating complex defects undetected during early walkthroughs and unit test.

\subsection{Evaluating the Accuracy of the Models}

We compare the results obtained using logistic regression and classification trees with those found using Optimized Set Reduction. The fully automated OSR process was used to generate the set of patterns partially presented in Section 3.3. For each modeling approach, a V-fold cross validation procedure was used $[\mathrm{BF}+84]$. Each pattern vector was successively removed from the dataset. The model was built using the remainder of the dataset and then used to predict the pattern vector extracted. The prediction is compared to the actual and this is repeated for each pattern vector in the dataset. Unless the available dataset is large, this validation method is preferable: this is an objective validation method (i.e., no arbitrarily selection of test sample) that allow model evaluations with a maximum number of observations.

The variable selection process used for building the regression models was a stepwise selection process with a predetermined selection criterion of $p=0.05$. Dummy variables [DG84] were created in order to deal with discrete explanatory variables. Principal components [DG84, HL 89, MK92] have been extracted and used in an attempt to optimize the accuracy of the regression models. Two regression models were built. The first one is based exclusively on the original explanatory variables. The second one uses, as explanatory variables, the generated principal components which are linear functions of the 
original explanatory variables, where each is orthogonal with respect to the others. With respect to classification trees, the algorithm provided by the S-PLUS system [S92] was used and the parameters controlling the tree construction were tuned in order to get optimal accuracies. However, this process was quite tedious since no guideline or rational exists for tuning these parameters despite the great instability of the generated trees.

When comparing modeling techniques with respect to identifying high risk components, two different evaluation parameters must be considered simultaneously. Assume that when a high risk component is identified, a remedial action is taken during the testing phase (e.g., more expensive and more effective code reading technique) and that the benefit of this remedial action is validated and quantifiable. We have to consider the completeness of the model (i.e., the percentage of high risk components identified by the model. The benefit of this remedial action on the development process quality will be a function of completeness since the larger the number of high risk components identified, the higher the error detection rate. Also, the correctness of the model (i.e., the percentage of components identified as high risk that are actually of high risk) allows the user to quantify the waste of resources due to the unnecessary applications of remedial actions.

Table 1 shows these two parameters for logistic regression, classification trees and Optimized Set Reduction. OSR appears to be more accurate than both logistic regression and classification trees with respect to all the criteria considered. We conclude that the benefits of the remedial actions taken when identifying high risk components are increased using OSR. These results seems to indicate an improvement of the OSR algorithm when compared with the earlier version presented in [BBH92] where there was no significant accuracy differences when compared with logistic regression.

The results shown in Table 1 have been obtained following the classification rules below:

- Logistic regression: if the calculated probability of a component belonging to the high risk class was below 0.5 , the low risk class was selected. Otherwise, the high risk class was selected.

- Classification trees: The risk class was selected based upon the proportion of non-faulty and faulty components in the matching tree leaf.

- OSR: For a given component, all the significantly reliable extracted patterns were considered for performing the classification. If those patterns all showed a high probability in the same risk class, then that class was selected. Otherwise, the risk class characterized by the pattern subset with the highest average pattern reliability was selected. If none of the extracted patterns happened to have a reliability significantly different from the random expected reliability, then the component was considered undetermined and thus classified randomly among the two risk classes.

By selecting biased classification rules (e.g., 0.4 decision boundary for logistic regression), the model completeness and correctness could be modified. However, when completeness increases, correctness decreases and vice-versa. The best correctness / completeness tradeoff depends on the particular application of the model. The results below were obtained using unbiased classification rules.

\begin{tabular}{|l|c|c|}
\hline Model & Correctness & Completeness \\
\hline Optimized Set Reduction & $92.11 \%(70 / 76)$ & $95.89 \%(70 / 73)$ \\
\hline Classification trees & $83.33 \%(60 / 72)$ & $82.19 \%(60 / 73)$ \\
\hline $\begin{array}{l}\text { Logistic regression without } \\
\text { Principal components }\end{array}$ & $76.56 \%(49 / 64)$ & $67.12 \%(49 / 73)$ \\
\hline $\begin{array}{l}\text { Logistic regression with } \\
\text { Principal components }\end{array}$ & $80.00 \%(52 / 65)$ & $71.23 \%(52 / 73)$ \\
\hline
\end{tabular}




\section{Table 1: Model Accuracies}

\subsection{OSR Patterns' Interpretations}

Comparison between the interpretability of logistic regression equations and OSR patterns may be found in [BTH93]. Issues associated with classification tree interpretation are discussed in [BBT92]. In this section, we illustrate and evaluate the interpretability of OSR patterns. Some of the patterns characterizing "data value / structure" errors will be described in order to illustrate the interpretation process in the OSR context. Patterns will be presented in a format facilitating their readability. Class boundaries will not be shown since they are not meaningful to the reader. Instead their corresponding quantiles on the explanatory variable range (in the appropriate context) will be used to describe predicates.

\subsubsection{Regression Equation}

The regression equation generated is as follows:

$\log \left(\frac{p}{1-p}\right)=0.337+0.0103$ SLOC -0.00107 LUADA -1.8274 LUFREUC

where $\mathrm{p}=\operatorname{Prob}($ component is high risk))

One of the main problems of logistic regression models with respect to their interpretation is the inherent instability of regression coefficients when the underlying assumptions of the model are not met (see [BTH93] for example and details). In some cases, looking at the correlation matrix may help avoid the problem when interpreting. Another related problem is that many good predictors were not selected by the stepwise selection process because of a strong correlation with already included parameters. In order to interpret the regression equations, the user has to look carefully at the correlation matrix and the regression equation in order to have some meaningful insight into the associations between explanatory variables and the dependent variable. Instability may be due to other causes like overinfluential data points (outliers) or interactions between explanatory variables [DG84, HL89].

We will demonstrate in the next paragraphs that, on our dataset, logistic regression does not extract a lot of the information which is provided by the data set. Some of the assumptions made in 3.2 .2 will be supported by the OSR patterns.

\subsubsection{Patterns for Data Value / Structure Errors}

The patterns listed below are the ones that seemed to confirm the assumptions stated in section 3.1. Our goal was not to make assumptions based on the generated patterns since this is a risky and dangerous approach to data analysis, i.e., exploratory data analysis. As a matter of fact, many of the generated patterns were not clearly understandable to us and did not fit in our list of assumptions. Generating interpretable patterns does not imply generating easy to understand patterns, which is due to the indirect and complex nature of some of the statistically significant associations extracted from our data sets. Moreover, since statistical models do not deal with causality, interpretation becomes an even more sensitive process.

Patterns are grouped according to the assumption they support. For each pattern presented, the entropy associated with each predicate (here singleton predicates) is shown just below the predicate itself. Patterns were generated entirely automatically without human intervention. As opposed to the classification tree approach [S92], no "tuning" of the algorithm was necessary since the parameters of the OSR algorithm are all intituively meaningful (e.g., user set statistical levels of significance for 
differentiating distributions) and can be set at once. The predicates' value intervals have been calculated automatically according to the procedure described in Section 2.2.1. This approach for handling predicate intervals automatically and dynamically (classes change in various contexts) gives more meaning to the interpretation of the OSR pattems. The first group of patterns is commented in detail in order to remind the reader about how to read these patterns. A definition of the metrics appearing in the patterns presented below is provided in Appendix I.

- Pattern Group 1: Complex code within a largely reused LUA (Assumptions 4 and 6)

$$
\begin{array}{ll}
\text { NDMAX } \in[52 \%-100 \%] & \wedge \\
H=0.89 & \text { LUFREUS } \in[0 \%-71 \%] \Rightarrow \text { High Risk } \\
& H=0.73 \\
\text { NDMAX } \in[52 \%-100 \%] & \wedge \text { LUFREUC } \in[0 \%-81 \%] \Rightarrow \text { High Risk } \\
H=0.89 & H=0.75
\end{array}
$$

Picking those components with a relatively small amount of reuse within the subset whose maximum statement nesting level is high implies a high probability that the component will be in the high risk class (i.e., to generate errors).

The individual impact of predicates (here all singletons) on the risk (i.e., probability to be in the high risk class) can be quantified by looking at the entropy variation they generate. NDMAX $\in[52 \%-$ $100 \%$ ] creates a variation of entropy of 0.11 (from 1.0 , the initial set entropy, to 0.89 ). In this context, a variation of entropy of 0.16 can be observed for LUFREUS $\in[0 \%-71 \%]$ (from 0.89 to 0.73 ). However, there is no strong evidence that the amount of reuse in a LUA is a high risk characteristic when NDMAX E [52\%-100\%]. In other words, this pattern group seems to indicate that architectural reuse pays off in terms of defect probability only in the context of complex components.

- Pattern Group 2: Large compilation units within a LUA with a high level of parametrization (Assumptions 2 and 6 ).

$$
\begin{aligned}
& (S L O C \in[57 \%-100 \%] \vee V \in[54 \%-100 \%]) \stackrel{\wedge}{\rightarrow} \underset{H=0.46}{\operatorname{LUPARPD} \in[53 \%-100 \%] \Rightarrow \text { Higb Risk }} \\
& \mathrm{H}=0.84
\end{aligned}
$$

LUPARPD is an indicator of the average program unit interface complexity within a particular LUA. This complexity seems even more difficult to handle for large components (i.e., large number of lines of code, operands and operators). Based on the process defined in section 2.4 , the reliability of this pattern has been assessed at $100 \%$ and appears to be significant at $\mathrm{RS}=0.06$. Since this data set is small, relatively few pattems show significances below 0.1 . Here again, there is no strong evidence that LUPARPD is a high risk characteristic in the context of small components. Large components with complex interfaces are risky while small components do not seem to be strongly affected.

- Pattern Group 3: Large and complex compilation units within a LUA containing high quantities of cascaded imports (Assumptions 3, 5 and 6).

$$
\begin{aligned}
& (\operatorname{SLOC} \in[57 \%-100 \%] \vee V \in[54 \%-100 \%]) \stackrel{\wedge}{\rightarrow} \operatorname{LUACTMAX} \in[64 \%-100 \%] \Rightarrow \text { High Risk } \\
& \mathrm{H}=0.84 \quad \mathrm{H}=0.0 \\
& \text { NDAVE }[65 \%-100 \%] \stackrel{\wedge}{\rightarrow} \text { LUCMDMP } \in[36 \%-100 \%] \Rightarrow \text { High Risk } \\
& \mathrm{H}=0.92 \quad \mathrm{H}=0.0
\end{aligned}
$$

Importing large quantities of cascaded declarations seems to significantly increase the risk of defects even in the context of large and/or complex components, i.e., large number of lines of code, operands 
and operators. Once again, small components do not seem to be affected.

In this pattern, the first predicate is an example of composite predicate and is the result of the merging process. Phi (i.e., the merging criterion) was fixed to 0.7 .

- Pattern Group 4: Complex compilation units in the context of a LUA that expors/imports large quantities of declarations towards other LUA's (Assumption 1, 5 and 6 ).

$$
\begin{aligned}
& \text { LUWBYCU } \in[79 \%-100 \%] \stackrel{\wedge}{\rightarrow} \text { DOBJ } \in[46 \%-100 \%] \Rightarrow \text { High Risk } \\
& \mathrm{H}=0.78 \quad \mathrm{H}=0.34 \\
& \text { LUWBYCU } \in[79 \%-100 \%] \stackrel{\wedge}{\rightarrow} \in[26 \%-100 \%] \Rightarrow \text { High Risk } \\
& \mathrm{H}=0.78 \quad \mathrm{H}=0.44 \\
& \text { LUCC } \in[93 \%-100 \%] \Rightarrow \text { High Risk } \\
& \mathrm{H}=0.0
\end{aligned}
$$

This pattern group seems to indicate that interfacing with other compilation units in order to export complex compilation unit (i.e., large number of declared / defined variables or a large cyclomatic complexity) shows a high defect risk. These patterns illustrates how the notion of context can play an important role when determining the impact of an explanatory variable. This shows that when one wants to validate assumptions, the answer may not be as simple as yes or no. In our particular example, most of the assumptions would not have been validated by simply looking at the regression model [CAP88].

- Pattern Group 5: When average statement nesting level is high, the "size" of the component is large and this component has an ALgorithmic / COMPutational functionality (according to the NASA SEL taxonomy), then there is a high probability that the component is high risk. Note that this is an example of the use of non-singleton predicates.

$$
\begin{aligned}
& \text { NDAV } \in[65 \% \cdot 100 \%] \stackrel{\wedge}{\rightarrow}(\text { ALCOMP YES } \wedge(\text { SLOC } \in[15 \%-100 \%] \vee \vee \in[19 \%-100 \%] \vee \\
& \begin{array}{ll}
H=0.92 & H=0.75
\end{array} \\
& \text { TOTASTMT } \in[23 \%-100 \%]))
\end{aligned}
$$

\section{Conclusions}

Five main conclusions can be drawn from this paper:

(1) Based on a rather small and incomplete data set, i.e., 146 Ada components, a completeness and a correctness above $90 \%$ has been obtained by using the OSR modeling process. If this level of accuracy is not sufficient, the user can tune the decisions boundary so he may increase either the correctness or completeness according to her/his specific needs.

(2) OSR Patterns appear to be more stable and interpretable structures than regression equations when the theoretical underlying assumptions are not met. Taking effective corrective actions is only possible when the impact of controllable factors on the parameters to be controlled (e.g., cost, quality) can be fully undersiood and quantified.

(3) OSR Patterns seem to generate a more complete set of information, i.e., validate more assumptions, than the logistic regression equation. This may be parially corrected by looking at the explanatory variable comelation matrix. However, this is an extremely tedious and not always 
helpful task, e.g., issues like interactions between explanatory variables are still not addressed.

(4) OSR classifications were found to be more accurate than logistic regression equations. This also confurms previous studies showing similar results for other kinds of applications [BBT92, BTH93]. Therefore, the Optimized Set Reduction approach seems to be a good alternative and/or complement to multivariate logistic regression in this application domain.

(5) OSR classifications were found to be more accurate than a classification tree. This also confirms earlier results we obtained on the datasets used in [BTH93] where classification trees were performing poorer than both logistic regression and OSR. These results seem to suggest that the classification tree structure, even though simple to generate and use, might be too simplistic for modeling complex artifacts such as high risk components.

From a more general perspective, the OSR approach is a data analysis framework that successfully integrates statistical and machine learning approaches in empirical modeling with respect to specific software engineering needs: it provides support for dealing with both partial information, model interpretation and is not based on a severely constraining set of hypotheses.

\section{Acknowledgments}

We would like to thank William Agresti, Frank McGarry and Jon Valett for their support in providing the data used in this analysis. Also, we would like to thank Sandro Morasca and William Thomas for their numerous comments that helped improve both the content and the form of this paper. 


\section{References}

[Agr90] A. Agresti, Categorical Data Analysis, John Wiley \& Sons, 1990.

[AES90] W. Agresti, W. Evanco, and M. Smith, "Early Experiences Building a Software Quality Prediction Model", Proceedings of the Fifieenth Annual Software Engineering Workshop, November,

[AE92] W. Agresti and W. Evanco, "Projecting Software Defects from, Analyzing Ada Designs", IEEE Trans. Software Eng., 18 (11), November, 1992 (to appear).

[AE+92] W. Agresti, W. Evanco, D. Murphy, W. Thomas, and B. Ulery, "Statistical Models for Ada Design Quality", Proceedings of the Fourth Software Quality Workshop, Alexandria Bay, New York,
August, 1992.

[BP84] V. Basili and B.T. Perricone, "Software Errors and Complexity: An Empirical Investigation," Communications of the ACM, vol. 27, no. 1, January 1984.

[Bas85] V. Basili, "Quantitative Evaluation of Software Methodology", Proceedings of the First Pan Pacific Computer Conference, Australia, July 1985.

[BR88] V. Basili and H. Rombach,"The TAME Project: Towards Improvement-Oriented Software Environments", IEEE Trans. Software Eng., 14 (6), June, 1988.

$[\mathrm{BF}+84] \mathrm{L}$. Breiman, J. Friedman, R. Olshen and C. Stone, Classification and Regression Trees, Wadsworth \& Brooks/Cole, Monterey, California, 1984.

[BP92] L. Briand and A. Porter, "An Alternative Modeling Approach for Predicting Eror Profiles in Ada Systems", EUROMETRICS' '92, European Conference on Quantitative Evaluation of Software and Systems, Brussels, Belgium, April 1992.

[BBH92] L. Briand, V. Basili and C. Hetmanski, "Providing an Empirical Basis for Optimizing the Verification and Testing Phases of Software Development", IEEE International Symposium on Software Reliability Engineering, North Carolina, October 1992.

[BTH93] L. Briand, W. Thomas and C. Hetmanski, "Modeling and Managing Risk early in Software Development", Intemational Conference on Sof tware Engineering, Maryland, May 1993

[BBT92] L. Briand, V. Basili and W. Thomas, "A Pattern Recognition Approach for Software Engineering Data Analysis", IEEE Trans. Software Eng., 18 (11), November, 1992. [CA88] D. Card and W. Agresti, "Measuring Software Design Complexity", Joumal of Systems and
Software, 8 (3), March, 1988.

[Cap88] J. Capon, "Statistics for the Social Sciences", Wadworth publishing company, 1988 [CE87] J. Cendrowska, "PRISM: An Algorithm for Inducing Modular Rules", Journal of Man-Machine
Studies, 27, pp.349. [DG84] W. Dillon and M. Goldstein, Multivariate Analysis: Methods and Applications, Wiley and
Sons, 1984.

[Dou87] D. Doubleday, "ASAP: An Ada Static Source Code Analyzer Program", TR-1895, Department 
of Computer Science, University of Maryland, August, 1987.

[EA92] W. Evanco and W. Agresti, "Statistical Representations and Analyses of Software", Proceedings of the 24th Symposium on the Interface of Computing Science and Statistics", College
Station, Texas, March, 1992.

[GKB87] J. Gannon, E. Katz, and V. Basili, "Measures for Ada Packages: An Initial Study", Communications of the ACM, 29 (7), July, 1986.

[HK81] S. Henry and D. Kafura, "Software Structure Metrics Based on Information Flow", IEEE Trans. Software Eng., 7 (5), September, 1981.

[HL89] D. Hosmer and S. Lemeshow, "Applied Logistic Regression", John Wiley \& sons, 1989

[M83] R. Michalski, "Theory and Methodology of Inductive Leaming." In R. Michalski, J. Carbonell \& T. Mitchell (Eds.), Machine learning (Vol. 1). Los Altos, CA: Morgan Kaufmann. [MK92] J. Munson and T. Khoshgoftaar, "The Detection of Fault-Prone Programs", IEEE Trans.
Software Eng., 18 (5), May, 1992.

[PA+82] H. Potier, J. Albin, R. Ferreol and A. Bilodeau, "Experiments with Computer Software , September, 1982.

[Qui86] J. Quinlan, "Induction of Decision Trees", Machine Leaming 1, Number I, 1986.

[Rom87] H. D. Rombach, "A Controlled Experiment on the Impact of Software Structure on Maintainability", IEEE Trans. Software Eng., 13 (3), March, 1987.

[S92] J. Chambers, T. Hastie, "Statistical Models in S", Wadsworth \& Brooks/Cole Advanced Books \& software, Pacific Grove, California

[SP88] R. Selby and A. Porter, "Leaming from Examples: Generation and Evaluation of Decision Trees for Software Resource Analysis", IEEE Trans. Software Eng., 14 (12), December, 1988. 


\section{Appendix I: Definitions of the metrics appearing in the paper}

\section{- Library Unit Aggregation (LUA) metrics:}

. LUACTMAX: total number of cascaded program unit declarations / maximum possible number of cascaded program unit declarations

. LUCMIMP: cascaded imported program unit declarations / direct imported program unit declarations

. LUWBYLU: number of library unit aggregations that contain a with statement to this compilation unit

. LUWBYCU: number of compilation units that contain a with statement to this compilation unit

. LUPARPD: number of parameters per program unit declaration in the LUA

. LUFREUC: fraction of old (reused verbatim) number of components in the LUA

. LUFREUS: fraction of old (reused verbatim) number of SLOC's in the LUA

. LUADA: number of Ada statements in the LUA

. LUCC: unique Imported declarations / unique exported declarations

\section{- Compilation unit metrics:}

. NDMAX: maximum statement nesting level

. NDAV: average statement nesting level

. SLOC: source lines of code

. V: Halstead's volume

.VG: cyclomatic complexity

. DOBJ: number of declared variables 


\section{Appendix II: Algorithms}

\section{The Merging Algorithm}

This merging process can be formalized using the following definitions and algorithms:

Recall the definition of predicate and composite predicate from section 2.1.1 and 2.4. Let cp represent a composite predicate. Then, we define:

- Definition A1: A context (C) is an ordered conjunction of composite predicates that defines a subset of pattern vectors PSS (i.e., PSS = SUBSET(PVS, C)).

- Definition A2: An association coefficient $a_{i j}^{c}$ is an assigned statistical degree of association between $c p_{i}$ and $c p_{j}$ in a data set PSS $=$ SUBSET(PVS, C). Let PSS $S_{i}=$ SUBSET $\left(P S S, c p_{i}\right)$ and let $P S S_{j}=\operatorname{SUBSET}\left(P S S, c_{\mathrm{j}}\right)$.

A two row-two column contingency table is defined as shown in Figure 5.

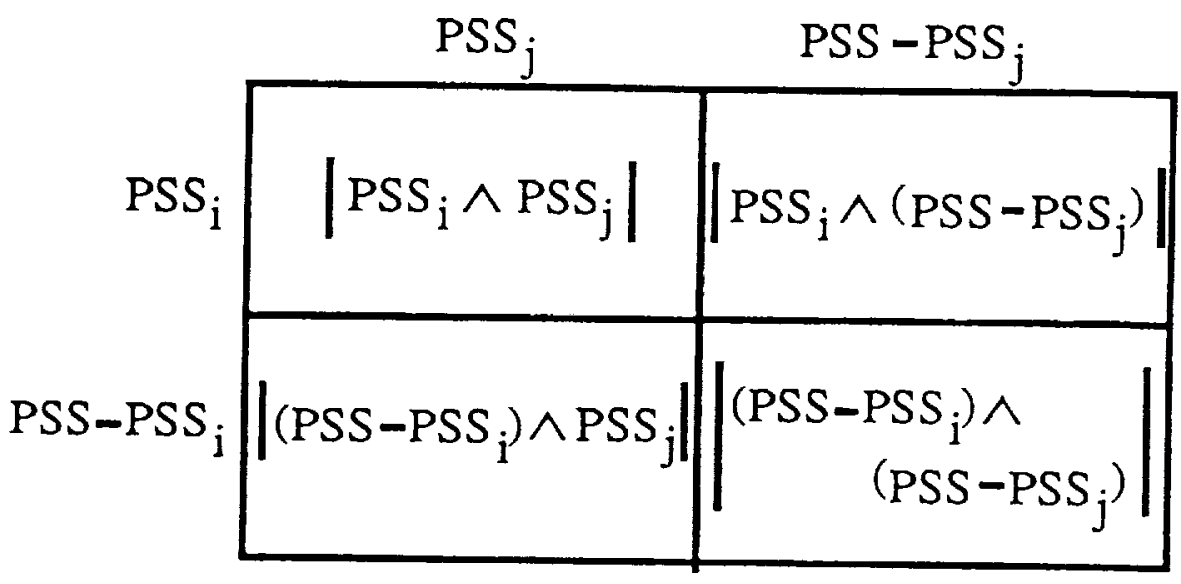

Figure 5: Predicate Association

Based on this table, a Chi-Square based statistic (Pearson's Phi), the degree of association between $\mathrm{cpi}$ and $\mathrm{CPj}_{\mathrm{j}}$ in PSS is calculated and assigned to $\mathrm{a}_{\mathrm{ij}}^{\mathrm{C}}$. Note that this association coefficient is calculated in the context of $C$ (i.e., PSS = SUBSET(PVS, C)) and therefore is only valid under $C$.

- Definition A3: An association matrix $A_{\mathrm{axp}}^{c}$ is a square matrix of association coefficients calculated under a context $C$, where the rows / columns are marked by composite predicates.

example: $A_{\mathrm{axo}}^{\mathrm{c}}$ contains all $\mathrm{a}_{\mathrm{ij}}^{\mathrm{c}}, \quad \mathrm{i}, \mathrm{j} \in\{1, \ldots, \mathrm{n}\}$

- Definition A4: Two composite predicates $c p_{i}$ and $c p j$ are said to be similar in the context of $C$ if $a_{i j}^{c}$ $\geq$ PHI (the minimal level of association defined by the user). This association will be denoted as 
$c p_{i}=c p_{j}$

- Definition A5: A predicate tree is a tree representation of the patterns generated when extracting the specific pattern set (SPS) process. As mentioned is Section 2.4, the SPS is a set of patterns representing the observed trends in the historical data set. It is expected that a significant number of these patterns will be duplicated or similar. This representation is a compact way of representing the SPS. Each path of a predicate tree represent a pattern (see Figure 6)

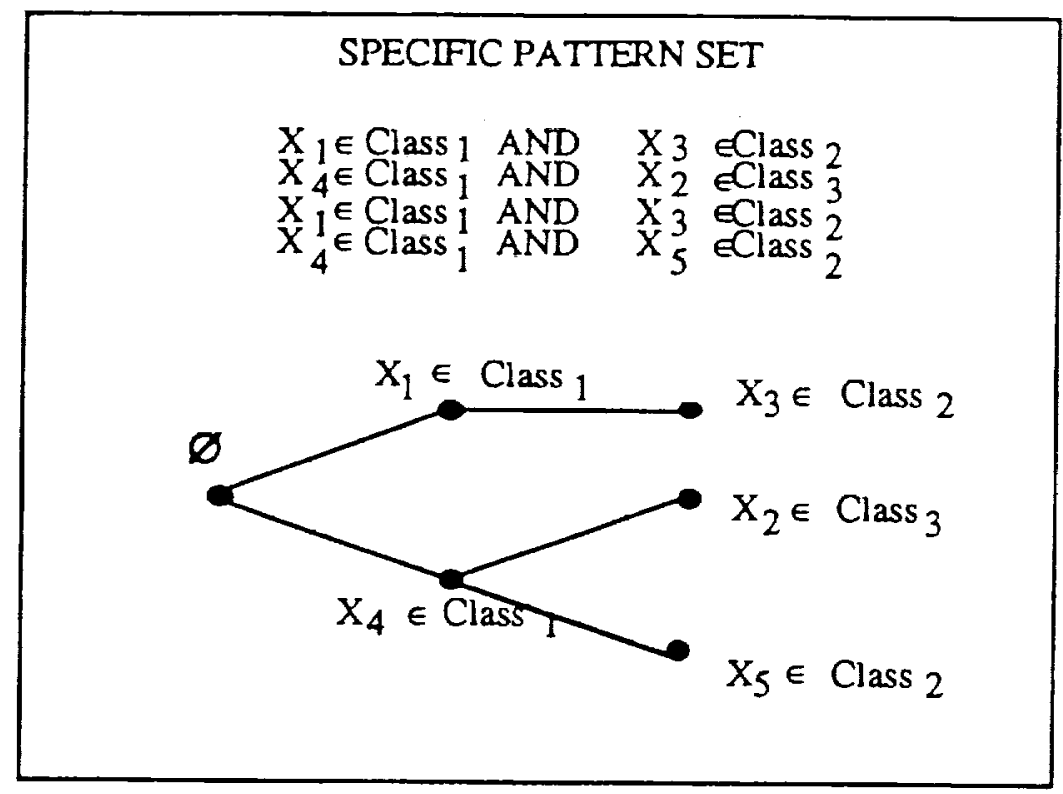

Figure 6: Predicate Tree

Note that in the above example, all of the predicates are singleton. This could represent a predicate tree which summarizes an OSR run. During the merging process, branches will be merged and composite predicates created at the nodes.

- Definition A6: Two composite predicates $c \mathrm{p}_{\mathrm{i}}, \mathrm{cp}_{\mathrm{j}}$ are said to be "mergable neighboring composite predicates" if the following conditions are fulfilled:

(1) There exist two predicates Pred $_{m}$ and Pred $_{n}$, where Pred $_{m}=\left(X_{i} \in\right.$ classik $_{i}$ and Pred $_{n}=\left(X_{i} \in\right.$

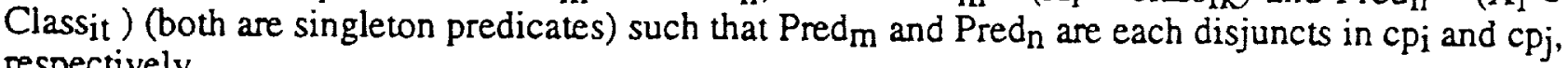
respectively.

(2) Classik and Classit are neighboring (or overlapping) classes on variable $X_{i}$ domain.

(3) $\mathrm{cpi}_{\mathrm{i}}$ and $\mathrm{cpj}$ yield similar distributions on the dependent variable range. (i.e., the level of significance of the two distributions being different is above $S$ (user defined)).

If these three conditions are true, then $\mathrm{MNCP}\left(\mathrm{cp}_{\mathrm{i}}, \mathrm{cPj}_{\mathrm{j}}, \mathrm{S}\right)$ is TRUE.

We can now define the merging algorithm as follows: 


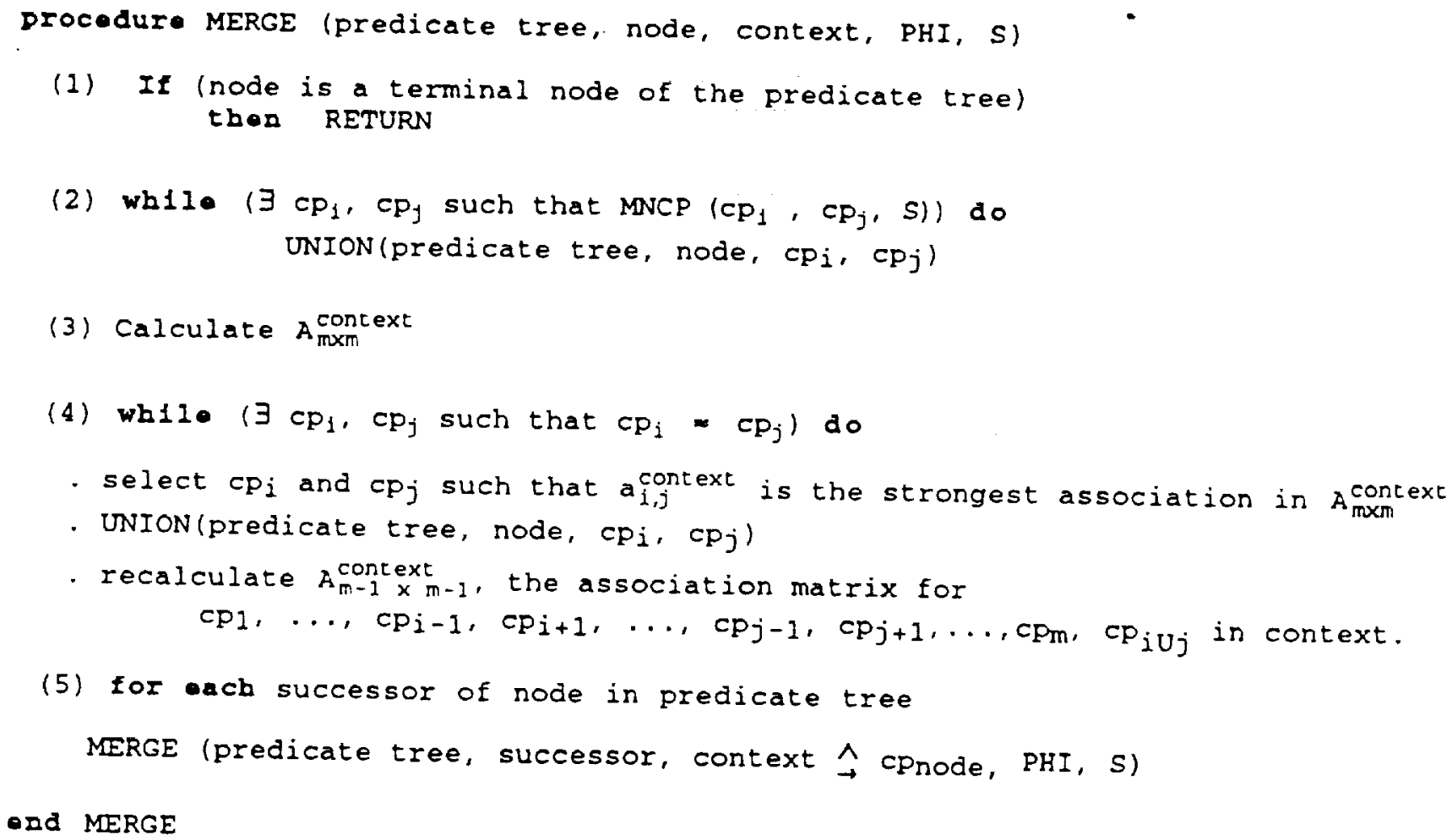

In step (4), a call is made to procedure UNION which is defined as follows:

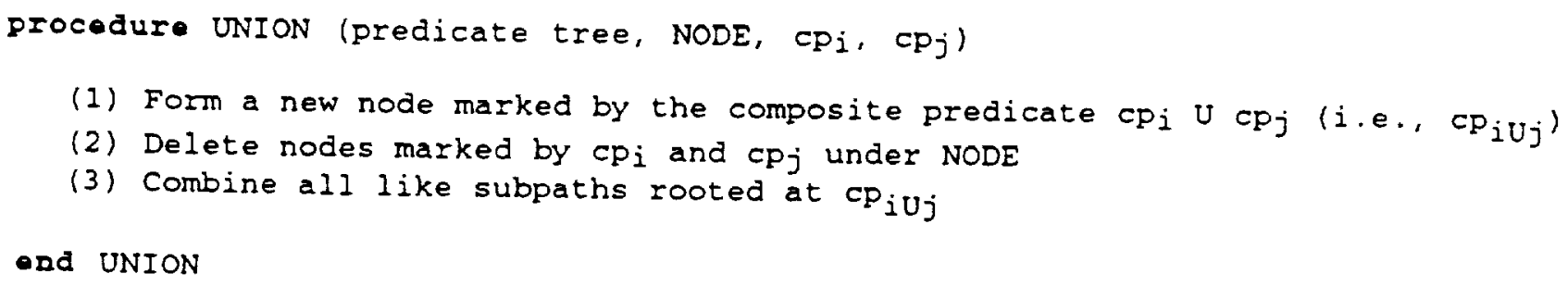

The merging process is initiated with the procedure call: MERGE (predicate tree, root, $\varnothing$, PHI, s) 


\section{Discretization Algorithm}

Procedure parameter definitions:

EV: the explanatory variable whose range is going to be discretized

DV: the dependent variable of the model to be built

. dataset: set of pattern vectors to be discretized along the scale of variable

- criterion: maximum level of significance accepted to recognize two distributions as different

. classes: the definiton of the intervals (classes) on variable's range, i.e., a set of pairs of boundaries

procedure DISCRETIZATION (dataset, EV, DV, criterion, classes)

(1) sort dataset elements in increasing order according to elements'variable values

(2) OPTIMAL_SPLIT(dataset, EV, DV, criterion, optimal_bound)

(3) If(dataset has actually been split in (2)) than

(3.1) update the definition of classes with newly calculated optimal bound

(3.2) extract two subsets sset1, sset2 of dataset where variable < optimal_bound and variable > optimal_bound, respectively

(3.3) DISCRETIZATION (ssetl, EV, DV, criterion, classes)

(3.4) DISCRETIZATION (sset1, EV, DV, criterion, classes) \}

\section{end DISCRETIZATION}

The procedure for splitting datasets may be defined as follows:

procedure OPTIMAL_SPLIT (dataset, EV, DV, criteria, optimal_bound)

for all data vectors $V_{i}$ in dataset (in sorted order)

l

Caso 1: there is a change in DV value but not in $E V$ value

( homogeneous = FALSE )

Case 2: there is a change in EV value (from EVVI to EVV2) and while EV values remained constant and equal to EVVI, homogeneous remained equal to TRUE

l

$/$ *

STEP I: calculate entropy of the distribution on the $D V$ range for the dataset subset lying in the interval strictly below EVV2 (SSET2)

STEP2: Calculate the level of significance of the difference in distribution between dataset and SSET2.

STEP3: If the the level of significance is below criterion and the entropy is below the minimal entropy calculated so far, then optimal_bound is assigned with EVV2 *I

Entropy $2=H($ SSET2, DV $)$

$10014023 L$ 


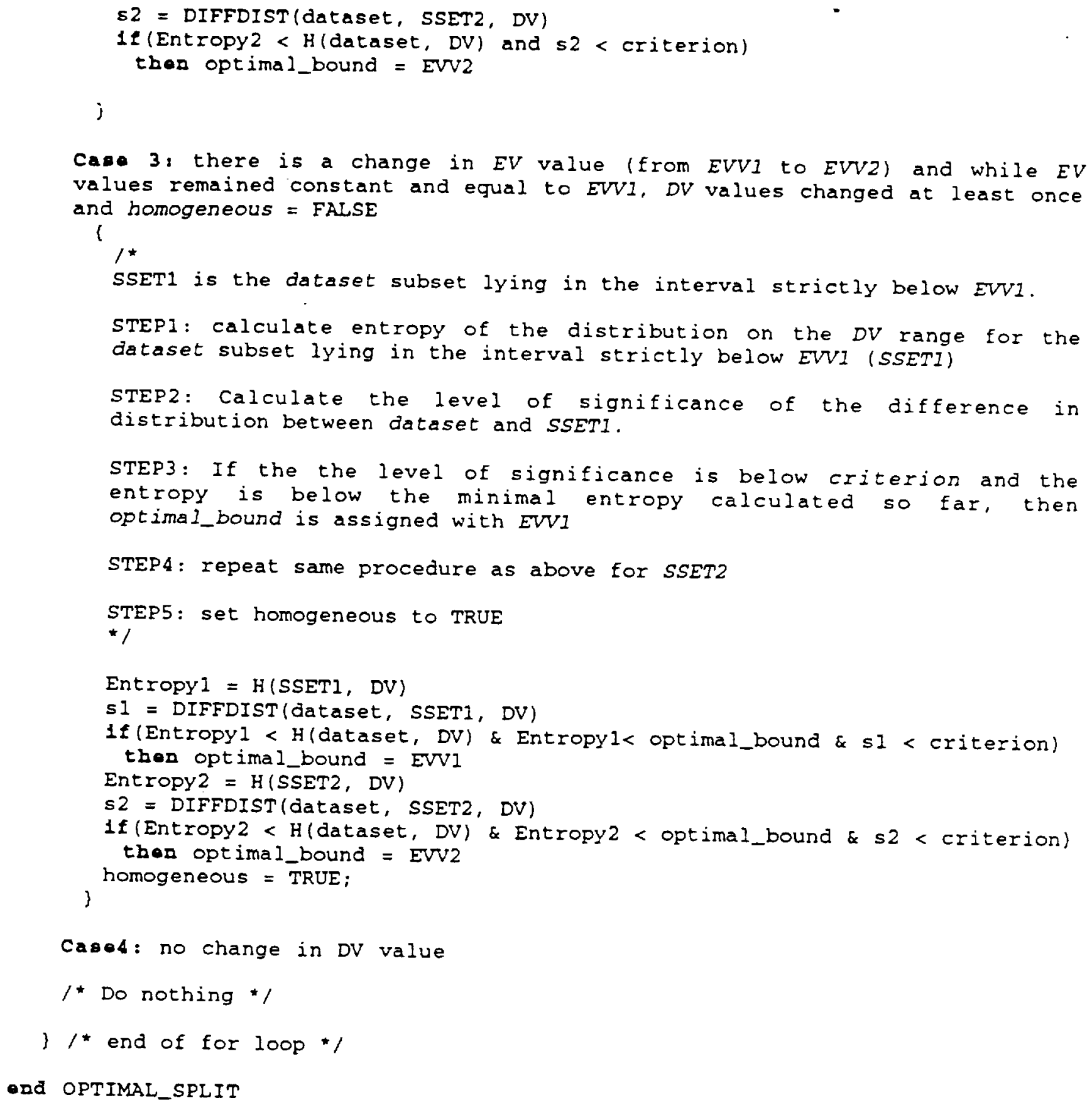


